\title{
Virtual Reality tools and statistical analysis for human movements simulation. Application to ergonomics optimization of workcells in the automotive industry
}

\author{
Stefania Spada ${ }^{\mathrm{a}, *}$, Fabrizio Sessa $^{\mathrm{b}}$ and Francesco Corato ${ }^{\mathrm{c}}$ \\ ${ }^{a}$ Manufacturing Engineering - Ergonomics, Fiat Group Automobiles, Torino, Italy. \\ b Manufacturing Engineering Southern Italy, Fiat Group Automobiles, Pomigliano d'Arco, Italy. \\ ${ }^{c}$ Faculty, of Engineering, SUN - Second University of Naples, Aversa (CE) Italy
}

\begin{abstract}
The paper show an application of Virtual Reality (VR) tools and Digital Human Modeling (DHM) methods to improve simulation of assembly tasks in order to achieve an ergonomic optimization of future workcells in car assembly plants. The goal is to perform the simulation in the very early phase of product and process development, when changes to project solutions are easy and costless. Generating synthetic motion with a Digital Human Modeling (DHM) tool that is ergonomically optimized for (eventually different) ergonomic indexes is a longstanding problem in the simulation analysis of ergonomics aspects of workcells. An ergonomic criterion which allows to classify a movement as a ergonomic or not, could be very useful to develop a new approach to synthetic motion generation in postural analysis. The application described is based on Motion Tracking techniques that allow to acquire human movements during a common assembly operation and to obtain the most important kinematic and information to characterize the movements. The results allow to evaluate ergonomics aspects of the synthetically generate motion on the base of statistical and wavelet analysis. By moving a 3D human model, it is possible to verify if the simulation of an assembly task is within an ergonomic criterion.
\end{abstract}

Keywords: Virtual simulation, motion capture, ergonomic classification, digital human modeling, statistical analysis

\section{Introduction}

In this paper a new method is presented to evaluate ergonomics content of a synthetic motion generated with a Digital Human Modeling (DHM) tool in order to achieve an ergonomic optimization of future workcells in car assembly plants. The result is the achievement of a synthetic motion that is ergonomically optimized for (eventually different) ergonomic indexes.

The application described in the next paragraphs is based on Motion Tracking techniques that allow to acquire human movements during a common assembly operation and to obtain the most important kinematic and information to characterize the movements. The results allow to evaluate ergonomics of the synthetically generate motion on the base of statistical and wavelet analysis. By moving a 3D human model, it is possible to verify if the simulation of an assembly task is within an ergonomic criterion (by means of the most important ergonomics methods). According to the result obtained, changes could be necessary to improve the project solution. By using the proposed approach, it is not more important to perform

*Corresponding author. E-mail: stefania.spada@fiat.com. Phone:+ 390110047134. 
again a new set of live motion capture sessions because the movements in the new situation can be obtained by a virtual human and easily evaluated if they match the ergonomics criterion. Again, ergonomics score allow new evaluation of the workcell. This process can be applied several times until the optimization result has been achieved.

The authors show results from a case study where they have used a Vicon motion tracking system and a classification analysis have been conducted on the movements captured in a series of gestures with the right arm-hand of many performers. Due to different styles of execution, the captured movements of different performers could constitute a limit for the process of movement classification. To this end, captured data will be "depersonalized" through a decomposition process based on wavelets in such a way to obtain a separation of the information present in the human motion: low frequencies (physical content), high frequencies (stylistic content).

The training phase of the classifier will be initially implemented as an EM (expectation-maximization) optimization of a Gaussian mixture model. The estimation of ergonomic score will be represented by the negative-log likelihood of the mixture. The motivation of a learn algorithm model parameters, like EM, is to produce a simple and efficient method of classification starting from a data set of motion characteristics. The performance of the classifier will be evaluated by the ROC curve (Receiver Operating Characteristic), analyzing the probability of obtaining truepositive and false-positive results. The results showing just over $82.5 \%$ confidence level seem to encourage our choice ergonomic criterion.

A key contribution of the paper is a new method to compute an ergonomics score for human motion. The measure will be useful to check if synthetically generated movement can be classified as ergonomic motion.

\section{Statistical Approach}

The used approach is based on the method realized by L. Ren et al. $[1,2]$ to check if synthetically generated movement can be classified as natural motions, but it differs from their method on a main aspect: we use a wavelet decomposition of motion captured data to eliminate the differences between movements due to different execution styles of the performers [3,], which could lead to a strong dependency of the classifier on the stylistic aspects of the movement data. In this first application of a statistical method to evalu- ate the ergonomic content of a human movement, we have based our analysis on postural ergonomics indexes only for the postures of the hand-arm system during performed trajectory.

A statistical ergonomic criterion should be useful for motions generated by DHM tools, in verifying that motion generation is ergonomically consistent with some ergonomic method. It is also necessary to verify that synthetically generated sub-task transition is within the space of those seen in ergonomic human motion.

For comparison purposes, it is assumed that a DHM model of arm-hand, performing some tasks presents a skeletal model and a number of degrees of freedom equal to the kinematical model of motion captured performer (for a discussion on the relationship between skeletal and kinematic model of the human body parts see [4]). However, due the statistical nature of the criterion, different hand kinematic models and different models of human body parts can be analyzed with the same criteria. For this, new training sets are needed.

To define our criterion we assume that data of an optically captured motion based on passive markers preserve the human movement ergonomics. This assumption is largely accepted, of course if the motion is "well captured", i.e.: (a) the calibration of the space of capture gives a small uncertainty on the $2 \mathrm{~d}$ view of markers on each camera; (b) the tracking of $3 \mathrm{~d}$ data has a high accuracy; (c) the skeletal and kinematical model are sufficient to reproduce the motion without any kind of perceptible alteration respect to the original one; (d) the algorithm used to calculate the joints and bones displacement of the skeletal model has a good precision; (e) there is not perceptible alteration of motion samples due to a massive editing of the captured data during a post processing stage, like motion re-sampling, jointing, blending, retargeting, time and space warping [5] [6] [7].

To collect ours ergonomic motion trials, we have used the optical motion capture system Vicon MX (C) by OMG inc. [8], with six two-megapixel camera; the space of capture was about $1.5 \mathrm{~m}^{2}$; the skeletal model of the right arm-hand has 25 degree of freedom.

\section{Ergonomic Motion}

The ergonomics of human motion depends on a multitude of issues and different indexes proposed in the literature assess: postures, forces, duration and 
frequency of task repetition, for all body parts with different criterion. Therefore, the purpose of the use of a statistical criterion to evaluate the ergonomics of a human activity in a workcell, eventually in cooperation with robots, is due to the ability to capture a multitude of complex behaviours that are ergonomics correct and many other movements that are not, to construct a good data training for the classifier.

In this paper we have only evaluated some postural aspects of movements, of course you can take into account criteria coming from different ergonomic indexes like [12]: RULA, EAWS, OCRA, etc., but this will need to rebuilt a new training set for the classifier where the data are evaluate using the new index. Although we believe that the overall ratings are only significant for each index, we have been verifying that local measures (on each bone or joint) give us some interesting results for posture evaluation especially if the ergonomics is evaluated for each class of motor task.

Based on motion capture data of human movement, we have worked on the characterization of kinematic aspects of human motion to define human movement as ergonomic one. In particular, movements of various performers will be captured and synthetic motions will be produced via DHM tool while doing the same task, to produce a first classification of movements in order to distinguish ergonomic movements and not-ergonomic ones.

\section{Development of the Classifier of Ergonomic Motion}

The training set of ergonomic classifier is constructed from data obtained from the positions of each degree of freedom for a set of sequences of homogeneous movements.

Based on these data, Mixtures of Gaussians (GM) are generated with $\mathrm{k}$ components ( $\mathrm{k}$ is experimentally determinate). Each component is a Gaussian, and the number of them, $\mathrm{k}$, that form the mixture should be no higher than the number of identified areas in which the data are grouped.

The GM is built by fitting the model parameters to data training. This procedure uses a method of supervised learning that exploits the statistical clustering algorithm partitioned EM. This algorithm derives the $\mathrm{k}$ components in which the data were grouped, then, it maximizes the likelihood, i.e. the probability that the data fall within the regions represented by the components of the mixture.

Given the hypothesis that the training set used to create the parametric model consists of sequences of ergonomic motion, the value of the likelihood of the mixture is the minimum value assumed for classifying a movement as ergonomic. The maximum value is represented by the probability $=1$. We will examine the negative of ergonomic logarithm of likelihood function, in order to have positive values in a scale ranging from zero to +infinite.

To benchmark the ergonomics of a new sequence of motion that is not part of the training set, will be calculated retrospectively the value of likelihood based on the mixture generated before. In summary, it is estimated the probability that the new sequence of kinematic data fall within the regions represented by the components of the GM. The likelihood value of the new sequence of motion is an estimate of the closeness of the new data to those of the parametric model previously learned. Operationally, if:

$\log \rho_{i}$ (with $i=1, \ldots \ldots, N$ ) is the negative logarithm of likelihood value of the ith DoF of the training set, and $-\log \pi_{i} \quad($ with $i=1, \ldots \ldots, N)$ is the negative logarithm of likelihood value of the ith DoF of the test motion; we verify if $-\log \pi_{\mathrm{i}} \leq-\log \rho_{\mathrm{i}}+\mathrm{T}$ (for any $\mathrm{i}=1, \ldots \ldots, \mathrm{N})$, with $\mathrm{T}$ an empirical tolerance value.

Hence, If the negative logarithm of new likelihood values (for each degrees of freedom) will fall in the range between 0 and $N \log \rho_{\text {i }}$ plus a tolerance value $T$, then the new sequence will be ergonomic.

The rise in the value of tolerance $\mathrm{T}$ that is added to the Nlog $\rho_{\mathrm{i}}$ of the GM increases the range of ergonomics, and therefore the number of sequences retained ergonomic, that they will be genuine or not, conversely, if we reduce it some ergonomic movements could be excluded. Tolerance Value $\mathrm{T}$ is identified during training of classifier empirically by use of ROC curve.

The "Receiver Operating Characteristic" allows the performance evaluation of a test based on threshold values. For quantitative tests, namely those that produce results in the form of discrete or continuous numerical variables, must be identified a threshold value or "cut off" which discriminates the results to be declared positive and negative. This allows us to categorize the range of all possible results into "positives" and "negatives".

The existence of a zone of overlap between the distributions of results of classification test determines 
uncertainty in understanding the results. For a complete discussion on R.O.C. see [9].

The performance of a classifier based on threshold can be measured by observing how many false negative it detect from the class of true-positive, and conversely how many false positive it detect from the class of true negatives, particularly the performance of our classifier is evaluated by observing how many of the sequences from ergonomic set it detect as not ergonomic and from the not-ergonomic sequences it detect as ergonomic. The ROC takes into account the relationship between true alarms and false alarms, analyzing the function that correlates the probability of a true-positive result in the class "ergonomic" (sensitivity) to the probability of obtaining a falsepositive result in the class "not-ergonomic" (specificity).

Using a system of coordinate axes, with the percentage of true positives in the ordinate and the percentage of false positives in the abscissa, we obtain the ROC curve by interpolation of the points made by each pair abscissa-ordinate. By varying the threshold you'll get different ROC curves.

To place a new sequence in the class "ergonomic" or "not-ergonomic" depends on the dimension of the AUC (Area Under Curve) plotted from the ROC curve. The AUC represents the probability that a new test does not give us any false results.

If a test does not return any false positives or false negatives, the AUC passes through the coordinates $(0$, 1) and its value turns out to be the area of the entire square bounded by the points of coordinates $(0,0),(0$, 1), (1.0) (1.1). In contrast, the ROC for a test without any informational value is represented by the diagonal passing through the origin, with $\mathrm{AUC}=0.5$.

To prove the ability of the classifier the test must verify that the area under the curve significantly exceeds the value of 0.5 . For the interpretation of AUC values, we can utilize the classification of the discriminative ability of a test proposed by Swets (1998):

- $\mathrm{AUC}=0.5$ - the test is not informative

- $0.5<\mathrm{AUC} \leq 0.7$ - inaccurate test

- $0.7<\mathrm{AUC} \leq 0.9$ - moderately accurate test

- $0.9<$ AUC $<1.0$ - very accurate test

- $\mathrm{AUC}=1.0$ - perfect test

\section{Experimental Result}

After explained the adopted model for the classification of a movement, let's now analyze the performance report the results obtained for a set of motion sequences. As we have explained previously for such assessment we will use the ROC curve, so that we can estimate the area under it (AUC). Experiments were carried out on two types of data: those related to the movement of a right arm-hand, acquired with our motion capture system, and those relating to the movements made by the use of a DHM tools.

\section{A. Training set for right arm motion classifier}

The database used for the model training is formed with fifty sequences of right arm-hand grasping. Each sequence is characterized by a particular grasp repeated over time. Each acquired movement consists of a number of frames that can vary between 500 and 2500; the arm-hand kinematical model has 25 degrees of freedom. The grasp tasks are performed by three different performers.

Through the fitting process of each movement sequence with the skeletal model we obtained measures of the angles of each joint.

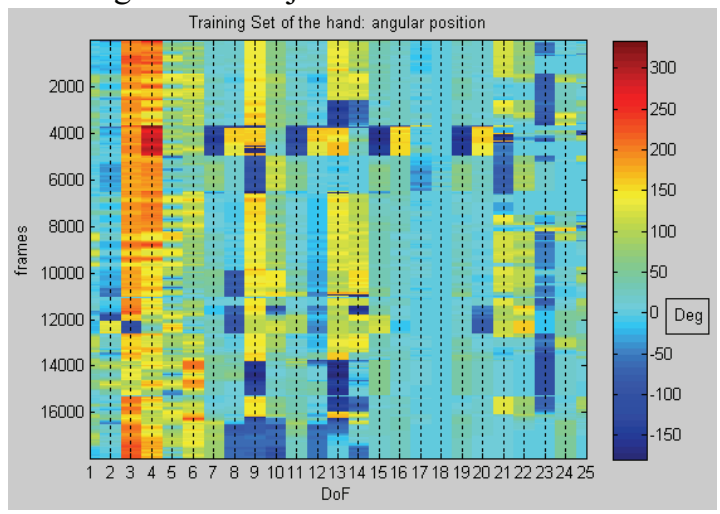

Fig. 1. Training set of angular positions of the arm-hand motion

The decision to generate Gaussian mixtures from the collected data, is due to the possibility of constructing a classifier of motion from a training set consisting of angular positions, regardless of the number of areas where data points are concentrated.

This set is evaluated to be ergonomic, any new sequence will be classified as ergonomic based on that set. 


\section{B. Construction of the ROC curve for angular po-} sition data

The construction of the ROC was conducted by examining 20 random sequences selected from ergonomic set and 20 not-ergonomic sequences, both for arm-hand movements. The abscissa of the graph represents the ratio of false positives and number of sequences considered not-ergonomic, while the ordinate is the ratio of true positives and number of ergonomic sequences tested. Specifically, selected 20 ergonomic movements, those that are recognized by ergonomic classification is to be the true positive, vice versa, selected 20 movements not-ergonomic, the number of those classified as ergonomic are the false positives.

To obtain the sequences of not-ergonomic motion has been used both a dedicated editing of the data captured than examples of not-ergonomic movements built using a DHM tool.

To evaluate the performance of the human-like motion classifier for hand motions we have tuned different threshold and tolerance value:

1) with empirical threshold value equal to the average score of the Gaussian plus a tolerance value equal to 1 and 2, we have:

- 20 true positive in 20 ergonomic;

- 12 false positive out of 20 not-ergonomic; the ROC curve is Fig. 2; the calculated AUC is equal to 0.675 ; being $0.5<\mathrm{AUC} \leq 0.7$ the test is not faithful enough;

2) with empirical threshold value equal to the average score of the Gaussian plus a tolerance value equal to 3 until 7, we have:

- 18 true positive in 20 ergonomic;

- 7 false positive out of 20 not-ergonomic;

the ROC curve is Fig. 3; the calculated AUC is equal to 0.775 ; being $0.7<\mathrm{AUC} \leq 0.9$ the test is faithful enough;

3) with empirical threshold value equal to the average score of the Gaussian plus a tolerance value equal to 8 until 12, we have:

- 18 true positive in 20 ergonomic;

- 14 false positive out of 20 not-ergonomic;

the ROC curve is Fig. 4; the calculated AUC is equal to 0.600 ; being $0.5<\mathrm{AUC} \leq 0.7$ the test is not faithful enough.

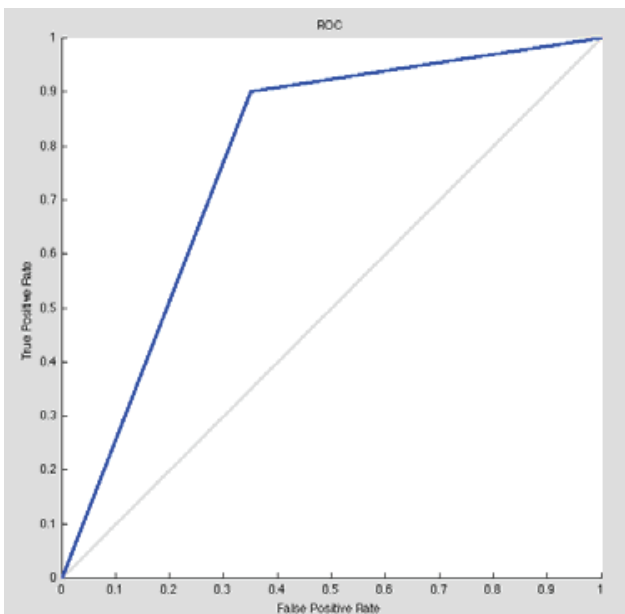

Fig. 2. ROC curve for Tolerance value equal 1 or 2 .

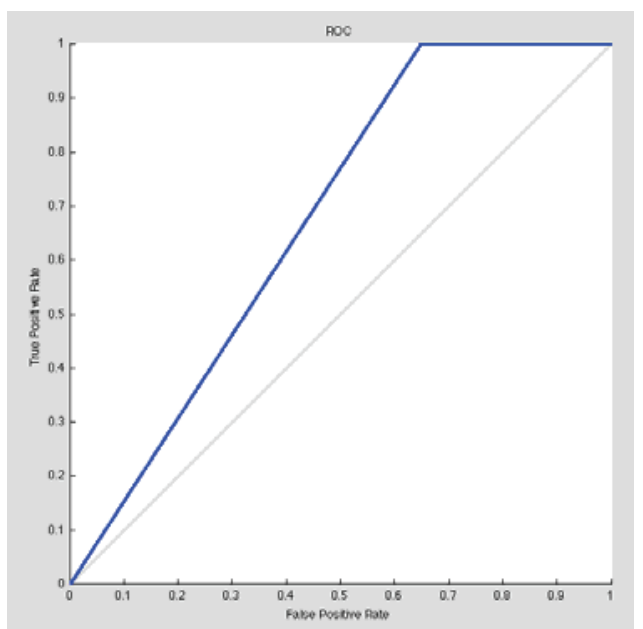

Fig. 3. ROC curve for Tolerance value equal 3 to 7.

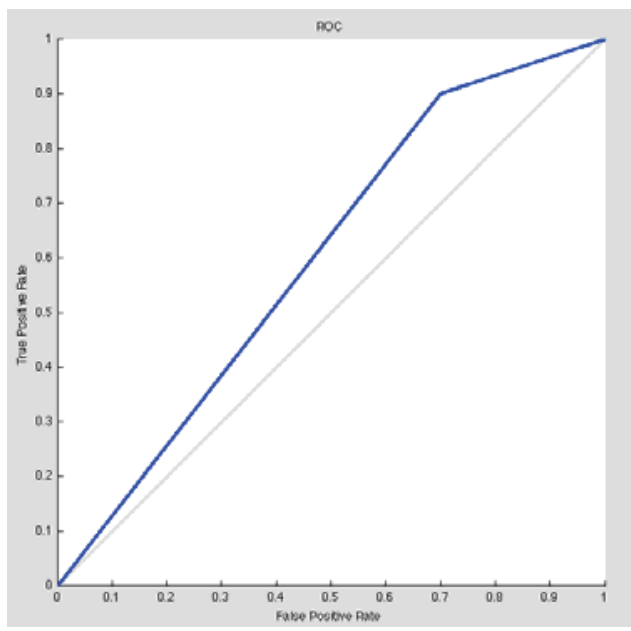

Fig. 4. ROC curve for Tolerance value equal 8 to 12 . 


\section{Training set for the hand motion classifiers based on DWT of angular acceleration data}

To improve the performance of the ergonomic motion classifier, a new training set is been realized using the discrete wavelet transforms (DWT) of angular data (for an introduction to wavelet analysis see $[10,11])$. The new data set will be constituted by the coefficients of approximation of a multi-level decomposition of angular position of joints.

We have tested different wavelet base function and the best performance was obtained with Haar function, at 2nd level of decompositon of angular position data. Although a $5^{\text {th }}$ level of decomposition give some interesting results on positional data, preserving the physical base of motion and separating the stylistic content, with a $2^{\text {nd }}$ level of decomposition we can note that approximation coefficients of the DWT for each DoF of training set give the best performance of the classifier.

Also in the case of DWT data, the construction of the ROC was conducted by examining 20 random sequences selected from ergonomic set and 20 notergonomic sequences, both for arm-hand movements. To evaluate the performance of the ergonomic motion classifier also in this case we have tuned different threshold and tolerance value:

1) with empirical threshold value equal to the average score of the Gaussian plus a tolerance value equal to 1 and 2 , we have:

- 11 true positive in 20 ergonomic;

- 0 false positive out of 20 not-ergonomic;

the ROC curve is Fig. 5; the calculated AUC is equal to 0.775 ; being $0.7<\mathrm{AUC} \leq 0.9$ the test is faithful enough;

2) with empirical threshold value equal to the average score of the Gaussian plus a tolerance value equal to 3 until 5, we have:

- 18 true positive in 20 ergonomic;

- 4 false positive out of 20 not-ergonomic;

the ROC curve is Fig. 6; the calculated AUC is equal to 0.825 ; being $0.7<\mathrm{AUC} \leq 0.9$, the test is faithful enough;

3 ) with empirical threshold value equal to the average score of the Gaussian plus a tolerance value equal to 4 until 10 , we have:

- 20 true positive in 20 ergonomic;

- 10 false positive out of 20 not-ergonomic;

the ROC curve is Fig.7; the calculated AUC is equal to 0.725 ; being $0.7<\mathrm{AUC} \leq 0.9$, the test is faithful enough.

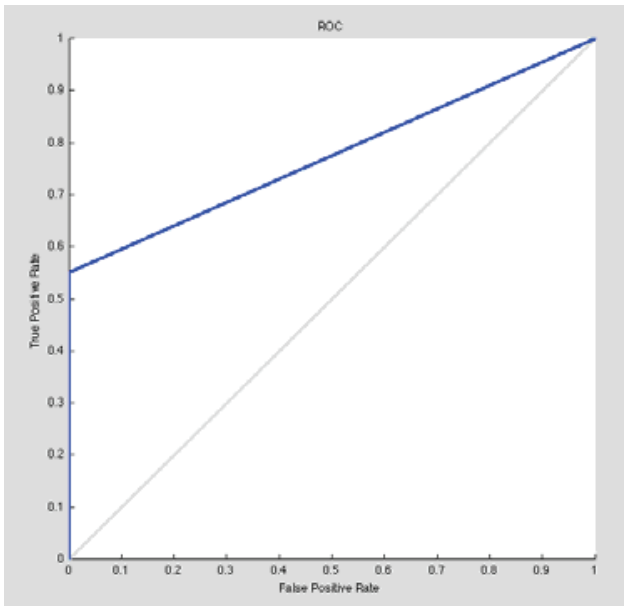

Fig. 5. ROC curve for Tolerance value equal 1 or 2.

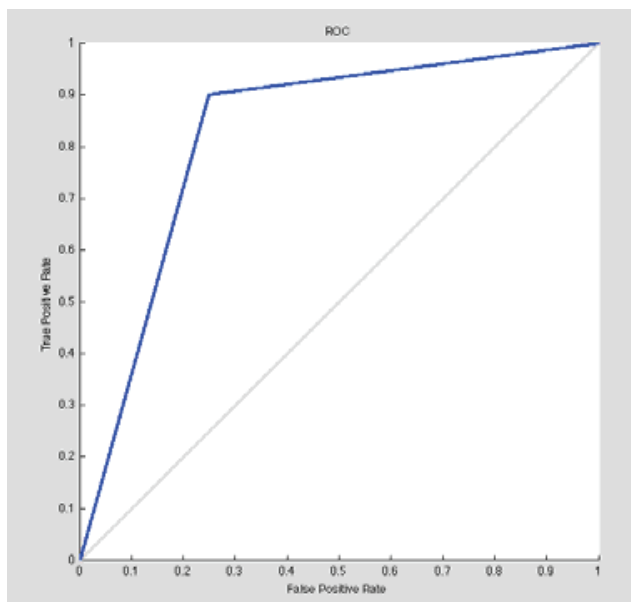

Fig. 6. ROC curve for Tolerance value equal 3 to 5 .

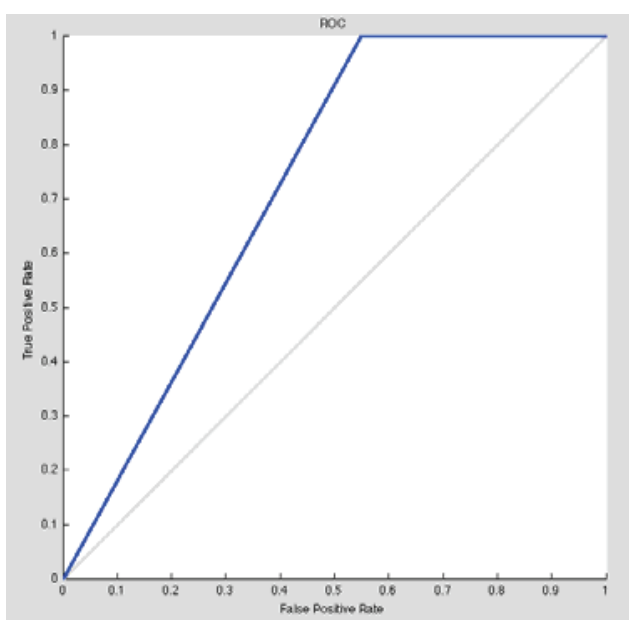

Fig. 7. ROC curve for Tolerance value equal 4 to 10 . 


\section{Conclusion}

To define a criterion for the ergonomics of armhand movements we have developed an ergonomic motion classifier. To do this we have used a parametric learning model, this method was chosen because is simple and effective. Specifically, through a process of supervised learning based on the EM clustering, Gaussian mixtures were generated from the angular positions of motion data. Furthermore, in order to compare different executions of the movement of different performers, we used a multilevel decomposition of motion data by multi-resolution analysis of discrete wavelet transforms. So, to generate the parametric model, were used only coefficients of approximation of the DWT of the angular positions.

The obtained results prove the validity of the model assumed, reaching good accuracy with particular values of the threshold. By use of the wavelets, the human-like classifier of hand motion has reached a value of AUC over $82 \%$, that is an improvement of the performance of the classifier without the use of DWT.

The classifier is very sensitive to the noise even few incorrect motion data could render the entire system unreliable and lead to wrong decisions.

We emphasize that the classifier for the recognition of ergonomic movements works well only on the motion data of the same type as those used for its training. Other type of movements could be classified as not-ergonomic although falling within the used ergonomic index.

\section{REFERENCES}

[1] L. Ren, A. Patrick, A. A. Efros, J. K. Hodgins, J. M. Rehg, “ A Data-Driven Approach to Quantifying Natural Human Motion”. ACM Transactions on Graphics,Volume 24, Issue 3 (July 2005). Proceedings of ACM SIGGRAPH 2005.

[2] L. Ren; "Statistical Analysis of Human Motion for Animation Computer" PhD thesis, Science Department School of Computer Science Carnegie Mellon University. 2006.

[3] T. Beth, I. Boesnach, M. Haimerl, J. Moldenhauer, K. Bois, V. Wank; "Characteristics in Human Motion - From Acquisition to Analysis", Humanoids, Germany 2003.

[4] F. Corato, P. Falco, M. Losh,, E. Maggio, J. Rainer, L.Villani "Original approaches to interpretation, learning and modelling, from the observation of human manipulation". Robotics Science and Systems Conference Seattle, Washington. 2009.

[5] F. Amalfitano, F. Corato, G. De Pietro "Motion Resampling for Optical Motion Captured Clips". Third International Conference on Human and Computer. Proc. Human and Computer 2000. Aizu- Tokyo, Japan.

[6] M. Gleicher "Comparing constraint-based motion editing methods". Graphical Models 63(2), 107-134. 2001.
[7] C. Rose, M. F., Cohen, B. Bodenheimer, "Verbs and adverbs: Multidimensional motion interpolation". IEEE Computer Graphics and Applications 18(5), 32-40. 1998.

[8] VICON MOTION SYSTEMS, 2005. http://www.vicon.com.

[9] T. Fawcett, "An introduction to ROC analysis", Pattern Recognition Letters, Vol. 27 , Issue 8. 2006.

[10] A. Grossmann, R. Kronland-Martinet and J. Morlet; "Reading and understanding continuous wavelet transforms"; Wavelets: Time- Frequency Methods and Phase Space, pp. 220, Springer-Verlag, Berlin 1989.

[11] S.G. Mallat, "A wavelet tour on signal processing"; Academic Press, 1999.

[12] J.T. Dennerlein, "Ergonomics/Musculoskeletal Issues", International Encyclopedia of Public Health, Elsevier Inc, 2008. 